\title{
La percepción de la autenticidad del destino cultural y su relación con la satisfacción y lealtad
}

Lilia Yolanda Noyola Aguilar Universidad de Extremadura lilia.noyola@valles.udg.mx

\author{
Ana María Campón Cerro \\ Universidad de Extremadura \\ amcampon@unex.es
}

Recibido: 15-01-2015

Aceptado: 11-04-2016

Keywords: authenticity; satisfaction; loyalty.

\begin{abstract}
The perception of the authenticity of the cultural destination and their relationship to satisfaction and loyalty. Today, despite the economic crisis that has emerged in recent years in different parts of the world, tourism industry it remains strong, growing and evolving. New trends in the travel industry are directed towards cultural tourism, with tourists interested in history, art and intend to live authentic and genuine experiences.
\end{abstract}

Therefore, the main objective of this study was to determine the perception of the authenticity of a cultural tourist destination and their relationship to satisfaction and loyalty. After analyzing the literature, a theoretical model which we propose to study the authenticity variables, satisfaction attributes, overall satisfaction and loyalty is performed through the study of measures of centrality, dispersion and correlation of variables. The results allow us to affirm that there is a positive correlation, although moderate, giving rise to further study this behavior, in order to generate more knowledge in this research and formulate strategies for managing relational marketing tourist destinations through cultural authenticity.
Palabras clave: autenticidad; satisfacción; lealtad.

\section{RESUMEN}

Hoy en día, a pesar a la crisis económica que ha surgido en los últimos tiempos en diferentes partes del mundo, el turismo se mantiene como una industria fuerte, en crecimiento y en constante evolución. Las nuevas tendencias en el sector de los viajes, se dirigen hacia el turismo cultural, con turistas interesados por la historia, el arte y con intención de vivir experiencias auténticas y genuinas. Por lo tanto, el objetivo principal de este trabajo es conocer la percepción de la autenticidad de un destino turístico cultural y su relación con la satisfacción y lealtad.

Una vez analizada la literatura, se realiza un modelo teórico donde se propone estudiar las variables autenticidad, satisfacción por atributos, satisfacción global y lealtad, a través del estudio de medidas de centralidad, de dispersión y correlación de las variables. Los resultados obtenidos nos permiten afirmar que existe una correlación positiva, aunque moderada de las variables. Con ello se puede continuar estudiando este comportamiento, con la finalidad de generar mayor número de conocimientos en esta línea de investigación y formular estrategias de marketing relacional para la gestión de los destinos turísticos culturales a través de la autenticidad. 


\section{INTRODUCCIÓN}

El turismo como rama de actividad económica, implica la participación de agentes sociales, públicos y privados, a nivel nacional, regional y local, siendo necesario establecer un vínculo de interacción, complementariedad y cooperación entre ellos y demás participantes claves del destino turístico como la población local, con el fin de construir relaciones satisfactorias con el turista a largo plazo.

A este enfoque se le conoce como marketing relacional, el cual según Kotler y Keller (2009) crea vínculos tanto económicos, como técnicos y sociales, al establecer relaciones mutuamente satisfactorias entre los consumidores, proveedores, distribuidores y otros socios de marketing.

En el turismo, el marketing relacional surge de manera natural (Quesada et al., 2009) y como en cualquier organización, el objetivo de los destinos turísticos es retener clientes a través de la satisfacción y la lealtad (Chi y Qu, 2008). Estas variables pueden medirse a través de indicadores que recojan la intención del cliente en repetir la compra o en este caso, de volver a visitar el sitio y recomendarlo a familiares y amigos (Martínez et al., 2009).

¿Pero qué elementos influyen para que un destino turístico genere satisfacción y lealtad en el turista?

Los atractivos turísticos constituyen un elemento crítico en la construcción de un destino, que se produce a través de la puesta en valor al resaltar las características del sitio (Almirón et al., 2006).

Actualmente, con la aparición de nuevos hábitos turísticos, la tendencia por el turismo cultural ha evolucionado y se ha consolidado cada vez más como un elemento favorecedor, tanto en destinos emergentes, como en mercados maduros y competitivos (Rodríguez y Fernández, 2009), con un perfil del turista interesado en conocer otras culturas, contemplando los recursos relacionados principalmente con la historia y el arte (Millet, 2011). Para Campón (2007:36) "los recursos culturales abarcan todas las manifestaciones del hombre, fruto del paso de los siglos y de la conservación de muchas tradiciones" tales como la arquitectura, la gastronomía, artesanía, folclore, etc., que forman parte de la motivación turística.

En este sentido, el patrimonio cultural depende de la importancia que se le otorgue a sus elementos para la recuperación, conservación, difusión y puesta en valor con el fin de originar interés en los visitantes. Es así, como uno de los principales retos para la gestión turística es mantener la autenticidad del destino, ya que al visitante le gusta creer en lo genuino y la experiencia vivida durante su visita, siendo ésta un elemento importante y diferenciador considerado a la hora de seleccionar el sitio a visitar (Vilar y Vidal, 2010).

Kolar y Zabkar (2009) argumentan que la autenticidad de un destino cultural juega un papel crucial que ayuda a comprender el comportamiento de los turistas. Esta autenticidad la describen Handler y Saxton (1988:2) como "una experiencia virgen, genuina, intocable y tradicional".

La autenticidad está presente como elemento diferenciador, donde el turista puede disfrutar de una experiencia única, imposible de encontrar en otros lugares, ya que es legítimo y exclusivo del destino. "La autenticidad no es creada para atraer, es utilizada para que realmente el turista vea al destino y sus recursos patrimoniales tales como son y los distinga de los demás, posibilitando a la oferta turística ventajas competitivas" (Vilar y Vidal, 2010:51).

Por lo tanto, el presente trabajo busca conocer el grado en que los turistas perciben la autenticidad de un destino turístico cultural y si este elemento influye para generar 
satisfacción y lealtad, haciendo posible su intención de repetir su visita y recomendarlo a otros.

En la Figura 1 se presenta el modelo conceptual formado por las variables y las relaciones que se pretenden estudiar.

FIGURA 1. Modelo teórico

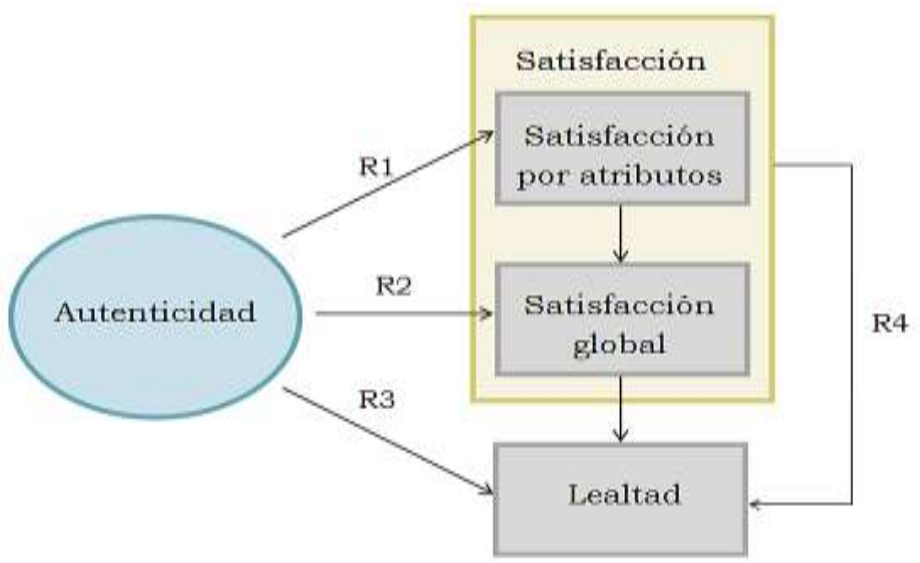

Fuente: Elaboración propia

\section{OBJETIVOS DE LA INVESTIGACIÓN}

Los destinos turísticos han sido estudiados desde diferentes perspectivas, utilizando diferentes tipos de variables, con el fin de conocer mejor el comportamiento del turista y proponer estrategias de gestión del destino.

\subsection{Objetivo general}

Es por ello que el objetivo principal de este trabajo de investigación es: conocer la relación entre la autenticidad percibida de un destino turístico cultural y la satisfacción y lealtad.

Con estos parámetros y con base a los fundamentos teóricos, se presentan 3 objetivos específicos.

\subsection{Objetivos especificos}

Objetivo 1: Analizar el efecto de la percepción de la autenticidad sobre la satisfacción de atributos y la satisfacción global ligada a la lealtad del destino turístico.

Objetivo 2: Establecer la relación entre las variables de autenticidad, satisfacción y lealtad de los destinos turísticos culturales.

Objetivo 3: Conocer las implicaciones que los resultados tienen tanto para el avance teórico en esta línea de investigación como para la gestión y practica de los destinos turísticos culturales.

\section{METODOLOGIA Y PLAN DE TRABAJO}

Para ésta investigación se realizó un muestreo no probabilístico de conveniencia eligiendo personas que se pueden disponer fácil y rápidamente, siendo un método utilizado en investigación exploratoria (Trespalacios et al., 2005). La población objeto de estudio está 
formado por turistas mayores de 18 años que visitan el Casco Antiguo de la ciudad de Cáceres, capital de la provincia homónima, situada al centro de la comunidad autónoma de Extremadura en España.

Como herramienta de investigación y recolección de información se ha recurrido a la encuesta personal directa, aplicado un cuestionario a los turistas durante su estancia en el destino seleccionado. En la encuesta personal directa existe un contacto directo entre el entrevistador y el entrevistado, siendo una técnica que permite un elevado porcentaje de respuesta, ya que el contacto directo ayuda a que exista mayor colaboración (Sanz, 2010).

Según Sanz (2010) la mayoria de los autores coinciden en que no existen reglas específicas para la elaboración del cuestionario, ya que lo importante es que cumpla con las expectativas que se plantean en la investigación. Por lo tanto nuestro cuestionario está estructurado en cinco bloques de la siguiente manera.

El primer bloque consta de una parte introductoria, donde se presenta el objetivo de la investigación. Se pregunta si es la primera visita al destino seleccionado, los días de estancia y con quién se realiza el viaje. Por último se cuestiona sobre el principal medio de información del destino.

El segundo bloque incluye las variables relacionadas con los motivos de viaje, medidos en una escala de Likert de 5 puntos ( $1=$ nada importante; $5=$ muy importante).

El segundo bloque está compuesto por preguntas relacionadas con la variable de la autenticidad del conjunto monumental ( 1 = nada de acuerdo; $5=$ muy de acuerdo).

El tercer bloque de preguntas aborda la variable de la satisfacción por atributos del destino turístico ( $1=$ malo; $5=$ excelente).

El cuarto bloque está conformado por las variables de la satisfacción global del destino y la lealtad ( 1 =nada de acuerdo; $5=$ muy de acuerdo).

Finalmente el quinto bloque se trata de preguntas demográficas que ayudan a la segmentación de la población, como son el sexo, la edad, nivel de estudios y el lugar de procedencia.

Según Trespalacios et al. (2005) para que los resultados de la investigación puedan extraerse de manera fidedigna, las preguntas del cuestionario deben ser elaboradas con cautela, a través de variables valoradas en función de una serie de escalas de medida delimitadas a priori. Por lo tanto para la escala de la medición de la motivación se han seleccionado 10 indicadores de la escala de Kolar y Zabkar (2009) y se han adaptado a los fines de la investigación, (ver Tabla 1).

TABLA 1. Indicadores de la variable motivación

\begin{tabular}{|c|c|c|c|}
\hline Dimensión & Estudios & Indicador Original & Indicador adaptado \\
\hline \multirow{5}{*}{ Motivación } & \multirow{5}{*}{$\begin{array}{l}\text { Kolar y } \\
\text { Zabkar } \\
(2009)\end{array}$} & Relax mentally & $\underset{\text { tranquilidad y relax }}{[\mathrm{MOT} 1]}$ Búsqueda $\quad \mathrm{de}$ \\
\hline & & $\begin{array}{cc}\text { Discover new } \\
\text { places and things }\end{array}$ & \begin{tabular}{lll}
\multicolumn{1}{l}{ [MOT2] } & Descubrir & nuevos \\
lugares & & \\
\end{tabular} \\
\hline & & $\begin{array}{l}\text { Increase } \\
\text { knowledge }\end{array}$ & [MOT3] Aprender cosas \\
\hline & & \multirow{2}{*}{$\begin{array}{l}\text { Have a good time } \\
\text { with friends }\end{array}$} & $\begin{array}{l}\text { [MOT4] Pasarlo bien con amigos } \\
\text { y/o familiares }\end{array}$ \\
\hline & & & $\begin{array}{l}\text { [MOT5] Visitar a familiares y/o } \\
\text { amigos }\end{array}$ \\
\hline
\end{tabular}


Noyola Aguilar, L. Y. y Campón Cerro, A. M. (2016): "La percepción de la autenticidad del destino cultural y su relación con la satisfacción y lealtad "



Fuente: Elaboración propia.

A su vez, para el estudio de la autenticidad se tomaron en cuenta los indicadores de Kolar y Zabkar (2009), por la similitud que tiene el trabajo de estos autores con el contexto de este estudio (ver Tabla 2)

TABLA 2. Indicadores de la variable autenticidad

\begin{tabular}{|c|c|c|c|}
\hline Dimensión & Estudios & Indicador Original & Indicador adaptado \\
\hline \multirow{10}{*}{ Autenticidad } & \multirow{10}{*}{$\begin{array}{l}\text { Kolar y } \\
\text { Zabkar } \\
\text { (2009) }\end{array}$} & \multicolumn{2}{|c|}{ Autenticidad basada en objetos } \\
\hline & & $\begin{array}{l}\text { The overall } \\
\text { architecture and impression } \\
\text { of the building inspired me. }\end{array}$ & $\begin{array}{lcr}\text { [AUT1] } & \text { El } & \text { conjunto } \\
\text { arquitectónico } & \text { de la } & \text { ciudad } \\
\text { monumental } & \text { me } & \text { inspira } \\
\text { autenticidad. } & & \\
\end{array}$ \\
\hline & & \multirow{2}{*}{$\begin{array}{l}\text { I liked the peculiarities } \\
\text { about the interior design/ } \\
\text { furnishings. }\end{array}$} & $\begin{array}{l}\text { [AUT2] Me parece } \\
\text { adecuada la conservación de la } \\
\text { ciudad monumental. }\end{array}$ \\
\hline & & & $\begin{array}{l}\text { [AUT3] } \text { Me } \quad \text { parece } \\
\text { adecuada la adaptación de la } \\
\text { ciudad monumental para el uso } \\
\text { turístico. }\end{array}$ \\
\hline & & $\begin{array}{l}\text { Iliked the way the site } \\
\text { blends with the attractive } \\
\text { landscape/ } \\
\text { historical ensemble/ town, } \\
\text { which offers many other } \\
\text { interesting places for } \\
\text { sightseeing. }\end{array}$ & 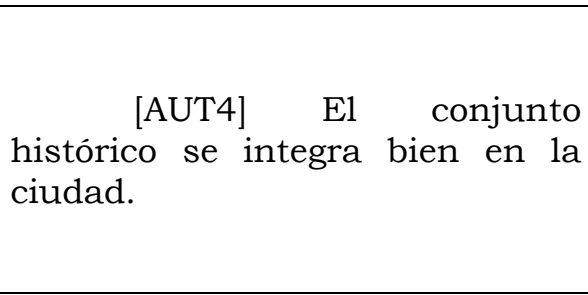 \\
\hline & & $\begin{array}{l}\text { I liked the information } \\
\text { about the site and found it } \\
\text { interesting. }\end{array}$ & $\begin{array}{l}\text { [AUT5] La información } \\
\text { sobre Cáceres y su patrimonio es } \\
\text { adecuada. }\end{array}$ \\
\hline & & \multicolumn{2}{|c|}{ Autenticidad existencial } \\
\hline & & \begin{tabular}{l}
\multicolumn{1}{c}{ I liked $\begin{array}{l}\text { special } \\
\text { events, }\end{array}$} \\
arrangements, \\
concerts, \\
connected to the site.
\end{tabular} & 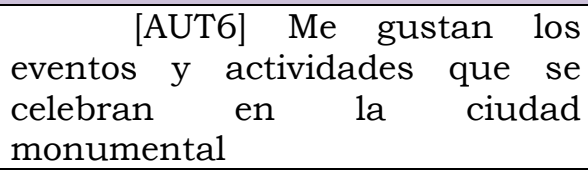 \\
\hline & & $\begin{array}{l}\text { During the visit I felt } \\
\text { the related history, legends } \\
\text { and historical personalities. }\end{array}$ & \multirow{2}{*}{$\begin{array}{l}\text { [AUT7] He disfrutado de } \\
\text { una experiencia única en la que } \\
\text { me he sentido transportado a la } \\
\text { época medieval. }\end{array}$} \\
\hline & & $\begin{array}{l}\text { I enjoyed the unique } \\
\text { religious and spiritual } \\
\text { experience. }\end{array}$ & \\
\hline
\end{tabular}


Noyola Aguilar, L. Y. y Campón Cerro, A. M. (2016): "La percepción de la autenticidad del destino cultural y su relación con la satisfacción y lealtad"

\begin{tabular}{|l|l|l|l|}
\hline & $\begin{array}{c}\text { I liked the calm and } \\
\text { peaceful atmosphere during } \\
\text { the visit. }\end{array}$ & $\begin{array}{c}\text { [AUT8] Me gusta el } \\
\text { ambiente relajado de la visita. }\end{array}$ \\
\hline
\end{tabular}

Fuente: Elaboración propia.

Para medir la satisfacción se tomó como base el estudio de Maroofi y Dehghan (2012) para los indicadores de satisfacción por atributos, así como la escala de Rodríguez del Bosque y San Martín (2008) para los indicadores de satisfacción global, incluyendo un indicador del estudio de Alegre y Cladera (2009)(ver Tabla 3)

TABLA 3. Indicadores de la variable satisfacción

\begin{tabular}{|c|c|c|c|}
\hline Dimensión & Estudios & Indicador Original & Indicador adaptado \\
\hline \multirow{16}{*}{$\begin{array}{l}\text { Satisfacción } \\
\text { de } \\
\text { atributos }\end{array}$} & \multirow{16}{*}{$\begin{array}{l}\text { Maroofi y } \\
\text { Dehghan } \\
(2012)\end{array}$} & \multirow{3}{*}{ Safety and security } & [SAT1] Accesibilidad \\
\hline & & & [SAT2] Limpieza \\
\hline & & & [SAT3] Seguridad \\
\hline & & $\begin{array}{l}\text { Peaceful and restful } \\
\text { atmosphere }\end{array}$ & $\begin{array}{l}\text { [SAT4] Ambiente tranquilo y } \\
\text { de descanso }\end{array}$ \\
\hline & & $\begin{array}{l}\text { Historic interests of } \\
\text { lodging }\end{array}$ & 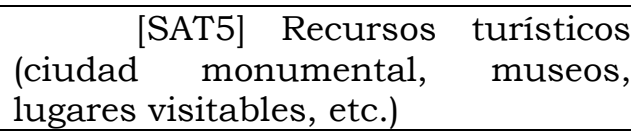 \\
\hline & & \multirow{2}{*}{$\begin{array}{l}\text { Service in lodging } \\
\text { facilities reasonable price of } \\
\text { meals }\end{array}$} & [SAT6] Alojamientos \\
\hline & & & 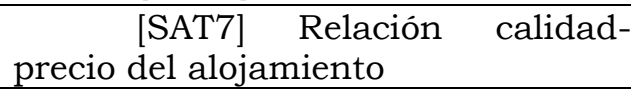 \\
\hline & & $\begin{array}{l}\text { Service } \\
\text { restaurants }\end{array}$ & [SAT8] Restaurantes \\
\hline & & $\begin{array}{l}\text { Quality of food } \\
\text { Reasonable price of }\end{array}$ & $\begin{array}{c}\text { [SAT9] } \\
\text { Relación } \\
\text { precio de los restaurantes }\end{array}$ \\
\hline & & $\begin{array}{l}\text { meals } \\
\text { Convenience }\end{array}$ & [SAT10] Riqu \\
\hline & & meals & gastronómica \\
\hline & & Variety of shops & $\begin{array}{l}\text { [SAT11] Tiendas para } \\
\text { comprar productos típicos y } \\
\text { souvernirs }\end{array}$ \\
\hline & & $\begin{array}{l}\text { Reasonable price of } \\
\text { merchandise }\end{array}$ & $\begin{array}{l}\text { [SAT12] Relación calidad- } \\
\text { precio de la venta de productos } \\
\text { típicos y souvernirs }\end{array}$ \\
\hline & & $\begin{array}{l}\text { Variety of cultural } \\
\text { options }\end{array}$ & $\begin{array}{cc}\text { [SAT13] Actividades } & y \\
\text { eventos culturales } & \end{array}$ \\
\hline & & Friendliness of local & $\begin{array}{l}\text { [SAT14] Trato al turista en } \\
\text { los establecimientos turísticos }\end{array}$ \\
\hline & & people & $\begin{array}{rlll}\text { [SAT15] } & \text { Amabilidad de la } \\
\text { población local } & & & \\
\end{array}$ \\
\hline \multirow{3}{*}{$\begin{array}{l}\text { Satisfacción } \\
\text { global }\end{array}$} & \multirow{3}{*}{\begin{tabular}{|l|}
\multicolumn{2}{r}{ Ro } \\
dríguez \\
del \\
Bosque y \\
San \\
Martín \\
$(2008)$
\end{tabular}} & I have really enjoyed & $\begin{array}{l}\text { [SAG1] He disfrutado de mi } \\
\text { viaje a Cáceres }\end{array}$ \\
\hline & & $\begin{array}{l}\text { My choice was a } \\
\text { wise one }\end{array}$ & $\begin{array}{l}\text { [SAG2]Venir a Cáceres fue } \\
\text { una buena elección }\end{array}$ \\
\hline & & $\begin{array}{l}\text { It is exactly what I } \\
\text { needed }\end{array}$ & $\begin{array}{l}\text { [SAG3]Visitar Cáceres es lo } \\
\text { que necesitaba }\end{array}$ \\
\hline
\end{tabular}


Noyola Aguilar, L. Y. y Campón Cerro, A. M. (2016): "La percepción de la autenticidad del destino cultural y su relación con la satisfacción y lealtad "

\begin{tabular}{|l|l|l|l|}
\hline $\begin{array}{l}\text { Alegre y } \\
\text { Cladera } \\
(2009)\end{array}$ & Overall satisfacción & $\begin{array}{c}\text { [SAG4]En general, me siento } \\
\text { satisfecho con la visita a Cáceres }\end{array}$ \\
\hline
\end{tabular}

Fuente: Elaboración propia.

Finalmente para la escala de la lealtad se han considerado los indicadores de la escala testada por Folgado et al. (2013)(ver Tabla 4).

TABLA 4. Indicadores de la variable lealtad

\begin{tabular}{|c|c|c|c|}
\hline Dimensión & Estudios & Indicador Original & Indicador adaptado \\
\hline \multirow{6}{*}{ Lealtad } & \multirow{6}{*}{$\begin{array}{l}\text { Folgado } \\
\text { et al. } \\
(2013)\end{array}$} & $\begin{array}{ccc}\mathrm{Me} & \text { gusta } & \text { visitar } \\
\text { Plasencia } & & \end{array}$ & $\begin{array}{l}\text { [LEA1] Me gusta visitar } \\
\text { Cáceres }\end{array}$ \\
\hline & & $\begin{array}{l}\text { Este destino seria mi } \\
\text { opción preferida para unas } \\
\text { vacaciones }\end{array}$ & $\begin{array}{l}\text { [LEA2] Este destino sería } \\
\text { mi primera opción para unas } \\
\text { vacaciones }\end{array}$ \\
\hline & & $\begin{array}{l}\text { En general, soy fiel a } \\
\text { este destino }\end{array}$ & $\begin{array}{l}\text { [LEA3] En general, soy fiel } \\
\text { a este destino }\end{array}$ \\
\hline & & $\begin{array}{l}\text { Recomendaría } a \\
\text { otras persona que visiten } \\
\text { Plasencia }\end{array}$ & $\begin{array}{c}\text { [LEA4] Recomendaré a } \\
\text { otras personas que visiten Cáceres }\end{array}$ \\
\hline & & $\begin{array}{c}\text { Repetiré mi visita a } \\
\text { Plasencia }\end{array}$ & $\begin{array}{l}\text { [LEA5] Repetiré mi visita a } \\
\text { Cáceres }\end{array}$ \\
\hline & & $\begin{array}{l}\text { Recomendaré } r \\
\text { experiencia a través de } \\
\text { Internet (webs, redes } \\
\text { sociales, blog) }\end{array}$ & $\begin{array}{l}\text { [LEA6] Recomendaré mi } \\
\text { experiencia a través de Internet } \\
\text { (webs, redes sociales, blog) }\end{array}$ \\
\hline
\end{tabular}

Fuente: Elaboración propia.

Las características de la investigación se resumen en la Tabla 5.

TABLA 5. Ficha técnica de estudio empírico

\begin{tabular}{|c|c|}
\hline Universo & Turistas mayores de 18 años \\
\hline Ámbito geográfico & $\begin{array}{c}\text { Cáceres. Localizaciones: Plaza Mayor de Cáceres, } \\
\text { Plaza Santa Maria, Plaza de San Jorge, Plaza de San } \\
\text { Mateo, Palacio de las Veletas, Torre de Sande. }\end{array}$ \\
\hline $\begin{array}{c}\text { Muestreo } \\
\text { Recogida } \quad \text { de }\end{array}$ & Encuesta personal asistida \\
\hline $\begin{array}{c}\text { Tamaño de la } \\
\text { información } \\
\text { muestra }\end{array}$ & 169 turistas \\
\hline $\begin{array}{c}\text { Fecha de trabajo } \\
\text { de campo }\end{array}$ & 17 de Abril-11 de Mayo de 2015 \\
\hline
\end{tabular}

Fuente: Elaboración propia. 
Para el análisis descriptivo de los datos se ha utilizado el programa estadístico SPSS Statistics Versión 19.0, para la evaluación de las variables a través de medidas de centralidad, de dispersión y correlación.

Para determinar la fiabilidad de las escalas de medida empleadas en esta investigación, se ha utilizado el método estadístico del alfa de Cronbach. Este coeficiente analiza la escala como una dimensión de su fiabilidad mediante el cálculo de la correlación entre los ítems de la escala. La interpretación de los resultados indica que si los diferentes ítems de una escala están midiendo una realidad común, las respuestas a estos ítems tendrian que presentar una elevada correlación entre sí. "La existencia de una baja correlación entre algunos de los ítems mostraría que algunas declaraciones de la escala no son medidas fiables del constructo" (Molina et al., 2008: 73).

Según Nunnally y Bernstein (1995) "en investigaciones de naturaleza exploratoria, el valor mínimo recomendado se sitúa en 0,70. La fiabilidad de las escalas de nuestro estudio se resume a continuación (ver Tabla 6).

TABLA 6. Análisis de la fiabilidad

\begin{tabular}{|l|c|c|}
\hline \multicolumn{1}{|c|}{ Variables } & No. de indicadores & Alfa de Cronbach \\
\hline Autenticidad & 8 & 0,780 \\
\hline Satisfacción por atributos & 15 & 0,886 \\
\hline Satisfacción global & 4 & 0,790 \\
\hline Lealtad & 6 & 0,849 \\
\hline
\end{tabular}

Fuente: Elaboración propia.

A partir de esta tabla se puede confirmar la fiabilidad de las escalas de autenticidad, satisfacción por atributos, satisfacción global y lealtad, obteniendo resultados superiores a 0,7 del coeficiente del Alfa de Cronbach.

Una vez comprobada la fiabilidad de las variables, procedemos al análisis de correlación, el cual se presenta a continuación.

\section{ANALISIS Y RESULTADOS}

Los resultados apuntan a un perfil de turista motivado por conocer el patrimonio histórico y artístico, la mayoría con edad entre los 26 y 45 años (47, 9\% en total) y con estudios universitarios en su mayoria $(84,0 \%)$. Lo cual nos habla de un visitante con mayor preparación e información encaminados a demandas de autenticidad de la experiencia y del destino, tratando de huir de los espacios turísticos que han sido concebidos para uso turístico masivo o consumo únicamente foráneo (Morales y Vela, 2009).

El 45,6\% ya habian visitado anteriormente Cáceres y el 55\% tuvieron como medio de información la recomendación de amigos y familiares, lo cual nos habla del alto grado de lealtad del turista, ya que según Martínez et al. (2009) la intención de recomprar el producto o de visitar nuevamente el destino turístico son indicadores que ayudan a medir el grado de lealtad de los clientes.

Para la evaluación de los estudios de correlación lineal bivariada entre las variables de autenticidad, satisfacción por atributos, satisfacción global y lealtad, con el fin de conocer el grado de relación entre dichas variables, se utilizó el coeficiente de Correlación de Pearson (R), el cual mide el grado de asociación lineal entre dos variables medidas en escala de intervalo o de razón, tomando valores entre -1 y 1 . Los valores de (R) próximos a 1 , indican una fuerte asociación lineal positiva. Valores de $(\mathrm{R})$ próximos a -1 , indican una 
Noyola Aguilar, L. Y. y Campón Cerro, A. M. (2016): "La percepción de la autenticidad del destino cultural y su relación con la satisfacción y lealtad "

fuerte asociación lineal negativa, mientras que los valores de $(\mathrm{R})$ próximos a 0 indicaran no asociación (Pedroza et al. 2007). A continuación se presentan los resultados obtenidos para cada correlación propuesta en el modelo teórico.

Los resultados nos indican que existe una correlación leve-moderada entre las variables autenticidad y satisfacción por atributos (R1) (ver Tabla 7).

TABLA 7. Análisis de correlación entre las variables de autenticidad y satisfacción por atributos. Relación 1 (R1).

\begin{tabular}{|c|c|c|c|c|c|c|c|c|c|c|c|c|c|c|c|}
\hline & SAT1 & SAT2 & SAT3 & SAT4 & SAT5 & SAT6 & SAT7 & SATB & SAT9 & SAT10 & SAT11 & SAT12 & SAT13 & SAT14 & SAT15 \\
\hline AUT1 & .003 & $33^{* *}$ & .136 & $85 \%$ & $.257^{\circ}$ & .133 & 67 & 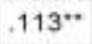 & .09 & 8 & .046 & 019 & 0.021 & $254^{* 1}$ & .104 \\
\hline AUT2 & 092 & $278^{*}$ & $265^{\prime \prime}$ & $382^{* *}$ & $270^{\prime \prime}$ & 097 & $315^{* \prime}$ & $267^{* *}$ & $.182^{*}$ & $.174^{\circ}$ & .161 & $.165^{*}$ & 0.148 & $342^{\prime \prime}$ & $316^{* *}$ \\
\hline AUT4 & $.173^{\circ}$ & $.303^{* *}$ & $.307^{* *}$ & $.317^{* 0}$ & $.403^{* t}$ & $244^{* *}$ & $.264^{* *}$ & $.185^{* *}$ & $191^{\circ}$ & $.220^{\prime \prime}$ & $.235^{* *}$ & $246^{* x}$ & $189^{\circ}$ & $381^{*}$ & $378^{*}$ \\
\hline AUTS & $239^{* *}$ & .116 & $.246^{*+}$ & $.345^{*}$ & $.439^{* t}$ & $304^{t+}$ & $.508^{* 0}$ & $238^{\circ *}$ & $.282^{n+}$ & $.172^{*}$ & $338^{* 4}$ & $385^{\star *}$ & $497^{\circ}$ & 412 & $395^{\text {th }}$ \\
\hline UT & $245 *$ & $.222^{* *}$ & $284^{*+}$ & $.436^{\circ}$ & $.518^{*}$ & .376 & .344 & .25 & .27 & .186 & .28 & .29 & .36 & 3 & .44 \\
\hline AUTB & $216^{* *}$ &, $331^{\cdots}$ & $.462^{* *}$ & $606^{*}$ & $.549^{* t}$ & $309^{* t}$ & $159^{\circ}$ & $.323^{* *}$ & $339^{* *}$ & $.324^{* *}$ & $.247^{*}$ & $219^{* *}$ & $.184^{\circ}$ & $.233^{*}$ & $.340^{* t}$ \\
\hline
\end{tabular}

** . La correlación es significativa al nivel 0,01 (bilateral)

* . La correlación es significativa al nivel 0,05 (bilateral)

Fuente: Elaboración propia.

Existe una correlación entre los indicadores [AUT6] "me gustan los eventos y actividades que se celebran en la ciudad monumental" y [SAT13] "actividades y eventos culturales" con un valor de 0,705. Lo cual nos dice que a mayor cantidad de eventos que se celebran en la ciudad monumental se logra mayor satisfacción por este atributo, siempre y cuando conserven la autenticidad del lugar, la cultura y las costumbres de la ciudad. Sin embargo la correlación entre [AUT1] "el conjunto arquitectónico de la ciudad monumental me inspira autenticidad" y nuevamente el indicador [SAT13] "actividades y eventos culturales" resulta negativa con un valor de $-0,021$ lo que explica que la autenticidad del conjunto arquitectónico de la ciudad monumental comienza a disminuir a medida que se potencian las actividades y eventos culturales. Este fenómeno podría relacionarse con eventos de gran magnitud que se celebran en estos sitios (Por ejemplo en la ciudad de Cáceres el Festival WOMAD o el Mercado Medieval de las Tres Culturas), ya que entre más gente asiste al evento, menos se aprecia la autenticidad de la ciudad.

A su vez, los indicadores [AUT8] "me gusta el ambiente relajado de la visita", el cual pertenece a la variable de autenticidad con carácter existencial, y el indicador [SAT4] "ambiente tranquilo y de descanso" de la variable de satisfacción por atributos, ambas con una correlación de 0,606, nos dice que el turista que busca una experiencia auténtica y escapando de la rutina considera el ambiente tranquilo como parte de su satisfacción.

En cuanto a los resultados de la correlación entre las variables autenticidad y satisfacción global (R2)(ver Tabla 8) encontramos que tiene valores bajos. Los resultados más significativos de la tabla es la correlación entre el indicador [AUT2] "me parece adecuada la conservación de la ciudad monumental" el cual esta enfocada a una autenticidad percibida en objetos o sitios, con los indicadores [SAG2] "venir a Cáceres fue una buena elección" con una correlación de 0,575 y [SAG1] "he disfrutado de mi viaje a Cáceres" con una correlación de 0,497 , lo cual nos indica que a mayor percepción de los 
turistas por la autenticidad basada en objetos aumenta su satisfacción global, considerando como buena elección el destino turistico cultural.

A su vez estos indicadores ([SAG1] y [SAG2]) tienen la menor correlación de la tabla con el indicador [AUT8] "Me gusta el ambiente relajado de la visita", con un valor de 0,232 para [SAG1] y de 0,275 para [SAG2], lo cual nos dice que no existe casi una correlación de que el ambiente relajado del sitio influya en las satisfación global.

TABLA 8. Análisis de correlación entre las variables de autenticidad y satisfacción global. Relación 2 (R2).

\begin{tabular}{|c|c|c|c|c|}
\hline & SAG1 & SAG2 & SAG3 & SAG4 \\
\hline AUT1 & $.388^{* *}$ & $.389^{* *}$ & $.336^{* *}$ & $.357^{* *}$ \\
\hline AUT2 & $.497^{k x}$ & $.575^{k k}$ & $.284^{+*}$ & $.366^{t+}$ \\
\hline AUT3 & $.400^{k k}$ & $.408^{k k}$ & $.229^{* t}$ & $.343^{t+}$ \\
\hline AUT4 & $.443^{*}$ & $487^{*}$ & $.283^{4 t}$ & $.362^{t+}$ \\
\hline AUT5 & $.304^{x}$ & $.401^{t+}$ & $.261^{\prime}$ & $.267^{\prime \prime}$ \\
\hline AUT6 & $.313^{* *}$ & $.289^{* *}$ & $.266^{*}$ & $.287^{*}$ \\
\hline AUT7 & $.389^{t+}$ & $.393^{* t}$ & $.422^{\mathrm{xt}}$ & $.353^{* *}$ \\
\hline AUTB & $.232^{* t}$ & $.275^{\text {to }}$ & $.311^{t+}$ & $.367^{*+}$ \\
\hline
\end{tabular}

** . La correlación es significativa al nivel 0,01

* La correlación es significativa al nivel 0,05

Fuente: Elaboración propia.

Los resultados del análisis de correlación entre las variables de autenticidad y lealtad (R3) (ver Tabla 9) nos indican que son leves. La correlación del indicador [AUT7] "he disfrutado de una experiencia única en la que me he sentido transportado a la época medieval" y los indicadores [LEA3] " en general, soy fiel a este destino "y [LEA4] "recomendare a otras personas que visiten Cáceres", con correlación para [LEA3] de 0,488 y para [LEA4] de 0,481 , lo cual nos dice que cuanto mayor sea la autenticidad percibida de la experiencia en el destino cultural mayor es el grado de lealtad y de recomendar el lugar a otras personas.

TABLA 9. Análisis de correlación entre las variables de autenticidad y lealtad. Relación 3 (R3).

\begin{tabular}{|l|l|l|l|l|l|l|}
\hline & LEA1 & LEA2 & LEA3 & LEA4 & LEA5 & LEA6 \\
\hline AUT1 & $.391^{* *}$ & .080 & -102 & $.306^{* *}$ & $.126^{* *}$ & $.196^{*}$ \\
\hline AUT2 & $.469^{* *}$ & $217^{* *}$ & $.172^{*}$ & $.406^{* *}$ & $.231^{* *}$ & $.331^{* *}$ \\
\hline AUT3 & $.312^{* *}$ & $-197^{* *}$ & $.205^{*}$ & $.292^{* *}$ & $.259^{* *}$ & $.260^{* *}$ \\
\hline AUT4 & $.356^{* *}$ & $.288^{* *}$ & $.220^{* *}$ & $.318^{* *}$ & $.276^{* *}$ & $.411^{* *}$ \\
\hline AUT5 & $.338^{* *}$ & $.386^{* *}$ & $.452^{* *}$ & $.391^{* *}$ & $.372^{* *}$ & $.455^{* *}$ \\
\hline AUT6 & $.216^{* *}$ & $.393^{* *}$ & $.385^{*}$ & $.320^{*}$ & $.320^{* *}$ & $.241^{* *}$ \\
\hline AUT7 & $.372^{* *}$ & $.425^{* *}$ & $.488^{* *}$ & $.481^{* *}$ & $.406^{* *}$ & $.577^{* *}$ \\
\hline AUT8 & $.340^{* *}$ & $286^{* *}$ & $267^{* *}$ & $.392^{* *}$ & $.379^{* *}$ & $.348^{* *}$
\end{tabular}

** . La correlación es significativa al nivel 0,01

* La correlación es significativa al nivel 0,05

Fuente: Elaboración propia. 
Por último la correlación entre las variables satisfacción por atributos, satisfacción global y lealtad nos muestra una relación leve-moderada.

Las correlaciones con valores más altas se presentan entre la satisfacción global y la lealtad, con los indicadores [SAG1] "he disfrutado de mi viaje a Cáceres", [SAG2] "venir a Cáceres fue buena elección" y [SAG4] "en general me siento satisfecho con la visita a Cáceres", cada una con correlación respecto al indicador [LEA1] "me gusta visitar Cáceres", con valores de 0,6. Lo cual indica que a mayor satisfacción global con el destino turístico cultural, mayor seria la lealtad hacia el sitio (ver Tabla 10).

TABLA 10. Análisis de correlación entre las variables de satisfacción por atributos, satisfacción global y lealtad. Relación 4 (R4).

\begin{tabular}{|c|c|c|c|c|c|c|c|c|c|c|}
\hline & SAG1 & SAG2 & SAG3 & SAG4 & LEA1 & LEA2 & LEA3 & LEA4 & LEA5 & LEA6 \\
\hline SAT1 & $.249^{* *}$ & $.207^{* *}$ & $.192^{*}$ & $.236^{* *}$ & $.239^{* *}$ & $.185^{*}$ & $.211^{\star *}$ & $.296^{\star *}$ & $.309^{\star *}$ & $.263^{* *}$ \\
\hline SAT2 & $.265^{\star *}$ & .137 & .149 & $.213^{* *}$ & $.276^{* *}$ & $.192^{\star}$ & .117 & $.419^{* \star}$ & $.225^{\star *}$ & $.278^{* *}$ \\
\hline SAT3 & $.332^{* *}$ & $.282^{\star *}$ & $.222^{* *}$ & $.296^{* *}$ & $.380^{* *}$ & .146 & $.190^{*}$ & $.490^{* \star}$ & $.320^{\star *}$ & $.403^{x \approx}$ \\
\hline SAT4 & $.425^{* x}$ & $.418^{* *}$ & $.306^{* *}$ & $.412^{* *}$ & $.496^{* x}$ & $281^{* \star}$ & $.308^{* *}$ & $.468^{\star \star}$ & $.338^{\star *}$ & $.395^{* *}$ \\
\hline SAT5 & $.290^{\star *}$ & $.376^{* t}$ & $.297^{\star *}$ & $.404^{\star \star x}$ & $.329^{* *}$ & $.314^{* *}$ & $.315^{\star \star}$ & $.479^{* x}$ & $.409^{* \star}$ & $.338^{* \star}$ \\
\hline SAT6 & $.190^{*}$ & .137 & $.212^{*}$ & $.199^{* \star}$ & $.196^{*}$ & $271^{* *}$ & $.306^{* *}$ & $283^{\star \star}$ & .129 & $.285^{t \star}$ \\
\hline AT7 & $.212^{* *}$ & $.274^{\star *}$ & $.216^{* *}$ & $.188^{*}$ & $.240^{* *}$ & $.333^{* \star}$ & $.413^{* *}$ & $.276^{\star \star}$ & $.332^{* *}$ & $.377^{* \star}$ \\
\hline SATB & .106 & $.238^{* t}$ & .133 & $.180^{\star}$ & .127 & $.224^{\star \star \star}$ & $.217^{\star}$ & $.280^{\star \pm}$ & $.187^{\star}$ & $.220^{* *}$ \\
\hline АТ9 & .130 & $.210^{* t}$ & $.184^{*}$ & $236^{* t}$ & $.268^{* *}$ & $.267^{\star \star}$ & $258^{* *}$ & $.353^{\star \star}$ & $.263^{\star \star *}$ & $.361^{* \star}$ \\
\hline AT10 & $.235^{\text {t* }}$ & $.241^{\star \star \star}$ & $.164^{*}$ & $.286^{\star \star *}$ & $.242^{* *}$ & $.136^{\star \star}$ & $.182^{\star}$ & $.295^{\text {t* }}$ & $.331^{\text {*t }}$ & .148 \\
\hline AT11 & .138 & $.222^{* \star}$ & $.206^{*}$ & $.199^{*}$ & $.158^{*}$ & $.239^{* *}$ & $.257^{\star \star}$ & $.191^{\star}$ & $.336^{\star *}$ & $.287^{* \star}$ \\
\hline AT12 & $.153^{\star}$ & .140 & $.213^{* *}$ & $.226^{\star \star}$ & $.151^{*}$ & $.296^{\star \star}$ & $.318^{* *}$ & $.247^{\star \star}$ & $.366^{\star *}$ & $.348^{* *}$ \\
\hline AT13 & $.177^{\star}$ & $205^{* *}$ & $.180^{* *}$ & $.184^{* \star}$ & .134 & $.367^{\star \star *}$ & $.341^{* *}$ & $274^{\star *}$ & $326^{\star *}$ & $.213^{* \star}$ \\
\hline AT14 & $.400^{* \approx}$ & $.362^{* \star}$ & $.370^{* *}$ & $.358^{* \star}$ & $.302^{* *}$ & $255^{\star \star}$ & $.278^{* *}$ & $.472^{\star \star}$ & $.357^{\star \star}$ & $.424^{x *}$ \\
\hline AT15 & $.379^{* *}$ & $.377^{\star \star}$ & $.311^{* *}$ & $.455^{\text {tz }}$ & $.429^{* \pm}$ & $.437^{\star \star *}$ & $.461^{* *}$ & $.395^{\star \star}$ & $.514^{\star \star}$ & $.495^{2 *}$ \\
\hline SAG1 & & $.689^{* \star}$ & $.488^{* *}$ & $.627^{\star \star}$ & $.613^{* *}$ & $.323^{\star \star}$ & $.358^{* *}$ & $.583^{\star \star}$ & $.416^{\star \star}$ & $.449^{* *}$ \\
\hline$A G 2$ & & & $.444^{x *}$ & $.530^{x *}$ & $.624^{* *}$ & $.333^{\star \star *}$ & $.387^{\star \star *}$ & $.595^{\star \star}$ & $.375^{\star \star}$ & $.426^{* *}$ \\
\hline 963 & & & & $.409^{* *}$ & $.435^{* *}$ & $.515^{\star x *}$ & $.518^{\star \star}$ & $.418^{* *}$ & $.388^{\star *}$ & $.514^{x *}$ \\
\hline AG4 & & & & & .628 & 370 & 381 & $.478^{* \star}$ & $.513^{\star \star \star}$ & 35 \\
\hline
\end{tabular}

** . La correlación es significativa al nivel 0,01

* . La correlación es significativa al nivel 0,05

Fuente: Elaboración propia.

Una vez evaluados los datos obtenidos del modelo teórico (R1, R2, R3 Y R4), se puede proceder a la discusión de los resultados, los cuales se detallan en el siguiente apartado.

\section{CONCLUSIONES E IMPLICACIONES}

Con la finalidad de dar respuesta a nuestro objetivo general y objetivos específicos, se realizó un modelo teórico que mide la correlación de la variable autenticidad, con las variables satisfacción por atributos, satisfacción global y lealtad. Así mismo, para obtener mayores datos sobre el perfil del turista se estudiaron las variables sociodemográficas y motivacionales.

Con los resultados obtenidos podemos constatar que existe una correlación positiva, aunque en una escala moderada entre las variables autenticidad y satisfacción por atributos.

Entre los principales resultados que se encontraron fueron que, a mayor número de actividades y eventos culturales que se celebran en el sitio auténtico, mayor es el grado de 
satisfacción por este atributo. Sin embargo, la autenticidad comienza a disminuir a medida que se incrementan estas actividades y eventos.

Por su parte los resultados nos señalan que el turista que busca la autenticidad existencial aprecia el ambiente tranquilo y de descanso. A mayor percepción de los turistas por la autenticidad basada en objetos y sitios, tienen mayor grado de satisfacción global, considerando su visita como una buena elección del destino y que han disfrutado de su estancia. Además, el haber vivido una experiencia única y auténtica en el destino cultural podría generar fidelidad y recomendarlo a familiares y amigos, ya que a mayor satisfacción global con el destino turístico cultural, mayor lealtad del turista.

Por ello, los gestores del destino turístico cultural podrian crear estrategias de marketing relacional enfocadas a buscar la satisfacción y lealtad del turista a través de elementos auténticos del destino, poniendo en valor sus recursos patrimoniales y realizar una buena promoción de los mismos, enfocada este perfil de turista. Sin embargo, se debe cuidar el no sobrepasar esta "explotación de la autenticidad" para que no pierda su valor genuino y se convierta en algo artificial.

Esta investigación se puede considerar como un estudio exploratorio inicial, dando pie a futuras líneas de investigación sobre la autenticidad de destinos turísticos culturales y las diferentes variables del marketing relacional.

En este mismo sentido, los estudios futuros podrian ser analizados con otros modelos estadísticos como el análisis de regresión y las ecuaciones estructurales, siendo necesario profundizar en las características del tipo de turista que busca la autenticidad en el destino turístico, a fin de generar estrategias de marketing relacional enfocadas a esta tipología de viajeros.

Por su parte sería interesante realizar el estudio de otros factores que acompañen a éstas variables como podrían ser necesidades, emociones y motivaciones en diferente escala.

Así como la relación de la misma sociedad receptora y sus características de estilo de vida y tradiciones ya que pueden estar implícitas en la preservación de la autenticidad del propio destino.

Por último, profundizar en los estudios que existen sobre los elementos que pueden hacer que se pierda la autenticidad de un destino turístico y conocer en qué escala podría influir negativamente en la satisfacción y lealtad de los turistas.

Con todo ello, se pretendería continuar avanzando en el análisis de la importancia de la autenticidad para la gestión de los destinos turísticos culturales y su importancia sobre la satisfacción y la lealtad, con los beneficios que eso conlleva para los mismos.

\section{REFERENCIAS BIBLIOGRAFICAS}

Alegre, J., \& Cladera, M. (2009): "Analysing the effect of satisfaction and previous visit son tourist intentions to return", European Journal of Marketing, 43:670-685.

Almirón, A., Bertoncello, R., \&Troncoso, C. A. (2006): “Turismo, patrimonio y territorio: Una discusión de sus relaciones a partir de casos de Argentina", Estudios y perspectivas en turismo, 15:101-124.

Campón Cerro, A. M. (2007): Cáceres como destino de turismo rural en mercados internacionales, Cáceres, Cámara de Comercio.

Chi, C. G. Q., \& Qu, H. (2008): "Examining the structural relationships of destination image, tourist satisfaction and destination loyalty: An integrated approach", Tourism management, 29:624-636.

Folgado Fernández, J. A., Di Clemente, E., Hernández Mogollón, J. M., \& Campón Cerro, A. M. (2013): "Influencia de la imagen del destino en el comportamiento del turista 
cultural. Un estudio empírico en la ciudad de Plasencia como destino de turismo cultural", Revista de Estudios Económicos y Empresariales, 95:91-114.

Handler, R., \& Saxton, W. (1988): "Dyssimulation: Reflexivity, narrative, and the quest for authenticity in "living history", Cultural Anthropology, 3:242-260.

Kotler, P., \& Keller, K. L. (2009): Dirección de marketing, Pearson education.

Kolar, T., \& Zabkar, V. (2009): "A consumer-based model of authenticity: An oxymoron or the foundation of cultural heritage marketing?”, Tourism Management, 31:652-664.

Maroofi, F., \& Dehghan, S. (2012): "Investigating the relationships of destination reflect, tourist satisfaction and destination loyalty", World Applied Sciences Journal, 19:1160-1173.

Martínez, F., Novello, S. y Murias, P. (2009): "Análisis de la lealtad de los turistas que visitan la ciudad de Santiago de Compostela”, Revista Galega de Economía, 18:1-15.

Millet, O. F. (2011): La imagen de un destino turístico como herramienta de marketing, Grupo EUMEDNET.

Morales, M. J., \& Vela, J. D. S. E. (2009): “La organización de eventos como estrategia identitaria y evocadora de imagen turística. Estudio de caso: Girona Temps de Flors”, COMITÉ EDITORIAL DIRECTOR: Agustín Santana Talavera, 7:73.

Molina, F. X., Martinez, M. T., Ares, M. A., \& Hoffman, V. E. (2008): La estructura y naturaleza del capital social en las aglomeraciones territoriales de empresas, Bilbao, Fundación BBVA.

Nunnally, Jum y Bernstein, Ira. (1995): Teoría Psicométrica, México, McGrawHill/Interamericana de México, S.A.

Pedroza, H., Dicovskyi, L., Bocchetto, R. M., Carneiro, M., Sparovek, G., Castagna Molina, M., \&Griffith, R. (2007): Sistema de análisis estadístico con SPSS (No. IICA-E1O1259). IICA, Managua (Nicarágua). INTA, Managua (Nicarágua).

Quesada, F. B. C., Hervé, A., \& Sánchez, M. D. S. A. (2009): "El sistema turístico en clave de marketing relacional: el factor relacional. Anuario jurídico y económico escurialense", 42:419-422.

Rodríguez Del Bosque, I. R., \& San Martin, H. (2008): “Tourist satisfaction a cognitive affective model", Annals of tourism research, 32:551-573.

Rodríguez, R. M., \& Fernández, J. I. P. (2009), "Desarrollo turístico y dinámica relacional: metodología de análisis para la gestión activa de destinos turísticos", Cuadernos de turismo, 23:173-194.

Sanz, M. J. M. (2010), Introducción a la investigación de mercados,ESIC Editorial.

Trespalacios, J. A., Bello, L., \& Vázquez, R. (2005), Investigación de mercados: Métodos de recogida y análisis de la información para la toma de decisiones en marketing, España, Paraninfo.

Vilar, L. G., \& Vidal, G. G. (2010), Fundamentos teóricos para una gestión turística del patrimonio cultural desde la perspectiva de la autenticidad, Eumed.

\section{HOW TO CITE THIS ARTICLE IN BIBLIOGRAPHIE}

Noyola Aguilar, L. Y. y Campón Cerro, A. M. (2016): "La percepción de la autenticidad del destino cultural y su relación con la satisfacción y lealtad" Rotur. Revista de Ocio y Turismo, 11: 65-76, ISSN-e 2695-6357 DOI: https://doi.org/10.17979rotur.2016.11.1.1781 